\title{
A national audit of the laboratory diagnosis of tuberculosis and other mycobacterial diseases within the United Kingdom
}

\author{
F A Drobniewski, B Watt, E G Smith, J G Magee, R Williams, J Holder, J Ostrowski
}

PHLS Mycobacterium Reference Unit, Dulwich Public Health Laboratory and Department of Microbiology, King's College School of Medicine, Dulwich Hospital, London SE22 8QF, UK

F A Drobniewski

J Holder

J Ostrowski

Scottish Mycobacteria Reference Laboratory, City Hospital, 51 Greenbank Drive, Edinburgh EH20 5SB, UK

B Watt

Regional Centre for Mycobacteriology, Birmingham Public Health Laboratory, Heartlands Hospital, Bordesley Green East, Birmingham B9 5ST, UK

E G Smith

Regional Centre for Mycobacteriology, Newcastle Public Health Laboratory, General Hospital, Westgate Road, Newcastle upon Tyne NE4 6BE, UK

J G Magee

Regional Centre for Mycobacteriology, Cardiff Public Health Laboratory, University Hospital of Wales,

Heath Park, Cardiff CF4 4XW, UK

$\mathrm{R}$ Williams

Correspondence to: Dr Drobniewski.

Accepted for publication 15 December 1998

\section{Abstract}

In order to audit United Kingdom laboratory diagnostic and reference services including novel molecular methods for tuberculosis, a questionnaire was sent to laboratories submitting specimens to the PHLS Mycobacterium Reference Unit (MRU) and regional centres and to the Scottish Mycobacteria Reference Laboratory (SMRL) in 1996-7. Nationally, 67.2\% of laboratories responded. Most UK laboratories were fully or conditionally CPA accredited and take part in the NEQAS proficiency scheme. On average only $3.3 \%$ of primary samples submitted for mycobacterial diagnosis in 1995 produced a mycobacterial culture from approximately half as many patients (that is, a mean of 1488 specimens producing 49 isolates from 23 patients). Potentially over 380000 specimens are processed for mycobacteria in the UK each year. The majority of laboratories use $4 \% \mathrm{NaOH} \pm$ NALC for specimen decontamination. Culture on solid media was used by most laboratories and $\mathbf{6 2 . 9 \%}$ also use liquid media. Most laboratories incubated cultures for eight weeks. Few laboratories use molecular diagnostic methods. Laboratories were most likely to use molecular methods for diagnosing tuberculous meningitis and for specimens from immunocompromised patients, although usage was strongly influenced by cost. Within England and Wales 43.9\% (47/107) and $56 \%(61 / 109)$ of laboratories wanted a rapid service for rifampicin resistance detection in $M$ tuberculosis from immunocompetent and immunocompromised patients, respectively. In regard to a tuberculous meningitis service, $80.5 \%$ (43I $112)$ and $84.3 \%(102 / 121)$ of laboratories wanted this service for immunocompetent and immunocompromised patients, respectively. The quality of reference services was rated as "very good"/"good" by $85.6 \%$ of respondents nationally. Rapid molecular amplification diagnostic services were established at the PHLS MRU for rifampicin drug resistance detection nationally and for tuberculous meningitis at the MRU.

(F Clin Pathol 1999;52:334-337)

Keywords: audit; Mycobacterium tuberculosis; molecular diagnosis; rifampicin resistance

Tuberculosis remains one of the commonest causes of adult morbidity and mortality in the world today. ${ }^{1-3}$ Although notifications in the industrialised world have fallen, Mycobacterium tuberculosis (MTB), the bacterium causing the disease, continues to produce nearly eight million new tuberculosis cases and three million deaths annually. ${ }^{1245}$ Notifications in Europe have increased in recent years ${ }^{16}$ and within the United Kingdom a steady decline reversed in 1987 and currently there are approximately 6000 notifications of clinical tuberculosis each year. $^{78}$

The PHLS Mycobacterium Reference Unit and Regional Centre for Mycobacteriology (MRU), London, PHLS regional centres for mycobacteriology (RCM) at Birmingham, Cardiff, and Newcastle, and the Mycobacteria Reference Laboratory, Scotland (SMRL) receive, identify, and perform drug susceptibility testing on over $95 \%$ of all mycobacteria cultured by hospital laboratories within the mainland United Kingdom. They also operate as diagnostic centres for their associated (and some additional) NHS Trusts.

\section{Methods}

Between August 1996 and May 1997, client laboratories of the PHLS MRU, the regional centres for mycobacteriology, and the SMRL were sent a detailed questionnaire. Questionnaires were prepared in EpiInfo 6 (Centres for Disease Control, Atlanta, Georgia, USA) at the MRU and piloted at two sites initially. The questionnaires built on earlier audits conducted by the SMRL and the Birmingham Regional Centre for Mycobacteriology. Replies were analysed in either EpiInfo 6 or Microsoft Excel.

The questionnaire requested information on the type of laboratory and containment facilities available, the numbers of samples received, number of mycobacteria cultured, methods used for microscopy and culture, internal and external quality control systems and the value 
Table 1 Methods used in United Kingdom laboratories for decontamination of samples for subsequent mycobacterial culture

\begin{tabular}{lrr}
\hline Method & Mean $(n=165)$ & $(\%)$ \\
\hline $4 \% \mathrm{NaOH}$ & 131 & $(79.4)$ \\
2\% NaOH + NALC & 4 & $(2.4)$ \\
$4 \% \mathrm{NaOH}+$ NALC & 10 & $(6.1)$ \\
Trisodium phosphate & 8 & $(4.8)$ \\
Sulphuric acid & 24 & $(14.5)$ \\
Oxalic acid & 24 & $(14.5)$ \\
Other & 7 & $(4.2)$ \\
\hline
\end{tabular}

$\mathrm{NaOH}$, sodium hydroxide; NALC, $\mathrm{N}$-acetyl cysteine.

of training and provision of standardised operating procedures by the MRU/RCM/SMRL. Laboratories were asked whether they currently used novel molecular amplification based systems, their views on the provision of these services by the PHLS or SMRL, and under what circumstances hospital laboratories felt they were likely to use these investigations. Finally laboratories were asked to comment on the performance of the MRU, SMRL, and regional centres for mycobacteriology with regard to the overall quality of the service provided, the accuracy and speed of the service, and the value of the scientific, technical, and medical advice given. Copies of the questionnaire are available from the authors.

Results are presented regionally, where relevant, and nationally for England and Wales or for the mainland United Kingdom for each of the questions asked. Not all questions were asked at every site.

\section{Results}

All responses were assessed as percentage of respondents answering each specific question. The overall United Kingdom response to the questionnaire was $67.2 \%(184 / 274)$.

\section{LABORATORY PROFILE}

In England and Wales the average number of samples in total received by participating laboratories was 115342 (range 101015 in northern England to 121731 in southern England). Nearly all laboratories had received either full or conditional clinical pathology accreditation (CPA) nationally (112/125 or $89.6 \%$ ), although this question was not posed in the north of England.

MYCOBACTERIAL DIAGNOSIS

Within England and Wales the average number of samples submitted for mycobacterial diagnosis was 1488 (range 1368 in northern
England to 1627 in southern England) which, in 1995, produced a mean of 49 mycobacterial isolates from a mean of 23 individual patients.

The majority of United Kingdom laboratories $(88.1 \%(163 / 185))$ take part in the NEQAS (National External Quality Assessment Scheme) quality control programme for microscopy and culture of mycobacteria. The national centres in London and Edinburgh asked their referring laboratories to record (in confidence) their average correct scores for mycobacterial QA (quality assurance) specimens for the preceding 12 samples, and $90.4 \%$ (75/83) scored maximum marks.

Interestingly, only $36.5 \%$ of United Kingdom laboratories (57/156) had a specific internal quality control system, which was reflected in the low number of respondents- $53.9 \%$ (57/156) — satisfied with their internal systems.

In the United Kingdom, $34.1 \%$ of laboratories (46/135) use Ziehl-Neelsen staining, $29.2 \%$ (38/130) use fluorescent staining, and $67.8 \%$ use both methods for microscopy of samples.

DECONTAMINATION OF SAMPLES

Various sample decontamination methods are employed in United Kingdom laboratories (table 1). Laboratories frequently employ different methods for different specimens and were able to indicate more than one choice. Samples are cultured on to a variety of solid or liquid culture media. Nearly all laboratories $(97.9 \%(138 / 141))$ responding within the United Kingdom use solid media, 96.3\% (131/ 136) buying it commercially. The number using a liquid culture medium, however, varies across the country, with $77.7 \%$ of laboratories (49/63) referring isolates to the MRU in London, $78.3 \%$ (18/23) referring to Birmingham Regional Centre for Mycobacteriology, 64.7\% (11/17) referring to SMRL, 39.4\% (13/33) to Newcastle Regional Centre for Mycobacteriology, and $26.7 \%(4 / 15)$ to Cardiff Regional Centre for Mycobacteriology. Overall $62.9 \%$ of respondents (95/151) use a liquid culture system.

Nationally, $46.3 \%$ of laboratories (76/164) incubate samples for eight weeks and $29.3 \%$ $(48 / 164)$ for 12 weeks at approximately $37^{\circ} \mathrm{C}$, and $32.1 \%(53 / 165)$ incubate some or all samples at $30^{\circ} \mathrm{C}$ or approximate skin temperature.

Contamination rates were actively monitored by only $41.3 \%$ of laboratories nationally (64/155). The contamination rates were

Table 2 Number of laboratories that would use a rapid molecular service to identify $M$ tuberculosis and isoniazid and rifampicin drug resistance in different clinical samples in immunocompetent patients, by region

\begin{tabular}{|c|c|c|c|c|c|}
\hline & London & Birmingham & Wales & Newcastle & Total \\
\hline Specimen type & $n(\%)$ & $n(\%)$ & $n(\%)$ & $n(\%)$ & Mean (\%) \\
\hline Smear $(+)$ sputum & $20 / 51(39.2)$ & $4 / 16(25.0)$ & $3 / 19(15.8)$ & $11 / 28(39.3)$ & $38 / 114(33.3)$ \\
\hline Smear $(-)$ sputum & $10 / 48(20.8)$ & $0 / 16(0)$ & $2 / 19(10.5)$ & $4 / 28(14.3)$ & $16 / 111(14.4)$ \\
\hline BAL & $25 / 48(52.1)$ & $4 / 14(28.6)$ & $5 / 19(26.3)$ & $9 / 28(32.1)$ & $43 / 109(39.4)$ \\
\hline CSF & $52 / 57(91.2)$ & $17 / 19(89.5)$ & $10 / 19(52.6)$ & $20 / 28(71.4)$ & $99 / 123(80.5)$ \\
\hline Lymph node & $24 / 50(48.0)$ & $7 / 15(46.7)$ & $4 / 19(21.1)$ & $8 / 28(28.6)$ & $43 / 112(38.4)$ \\
\hline Biopsy material & $31 / 52(59.6)$ & $10 / 17(58.8)$ & $2 / 19(10.5)$ & $9 / 28(32.1)$ & $52 / 116(44.8)$ \\
\hline Bone marrow & $30 / 52(57.7)$ & $7 / 15(46.7)$ & $7 / 19(36.8)$ & $10 / 28(35.7)$ & $54 / 114(47.4)$ \\
\hline Rifampicin drug resistance & $23 / 44(52.3)$ & $10 / 16(62.5)$ & $5 / 19(26.3)$ & $9 / 28(32.1)$ & $47 / 107(43.9)$ \\
\hline Isoniazid drug resistance & $20 / 44(45.5)$ & $8 / 16(50.0)$ & $5 / 19(26.3)$ & $9 / 28(32.1)$ & $42 / 107(39.3)$ \\
\hline
\end{tabular}

BAL, bronchoalveolar lavage; CSF, cerebrospinal fluid; (+), positive; (-), negative. 
Table 3 Number of laboratories that would use a rapid molecular service to identify $M$ tuberculosis and isoniazid and rifampicin drug resistance in different clinical samples in immunocompromised patients, by region

\begin{tabular}{llllll}
\hline Specimen type & $\begin{array}{l}\text { London } \\
n(\%)\end{array}$ & $\begin{array}{l}\text { Birmingham } \\
n(\%)\end{array}$ & $\begin{array}{l}\text { Wales } \\
n(\%)\end{array}$ & $\begin{array}{l}\text { Newcastle } \\
n(\%)\end{array}$ & $\begin{array}{l}\text { Total } \\
\text { Mean (\%) }\end{array}$ \\
\hline Smear (+) sputum & $37 / 54(68.5)$ & $10 / 18(55.6)$ & $7 / 19(36.8)$ & $18 / 28(64.3)$ & $72 / 119(60.5)$ \\
Smear (-) sputum & $23 / 53(43.2)$ & $8 / 17(47.1)$ & $7 / 19(36.8)$ & $9 / 28(32.1)$ & $47 / 117(40.2)$ \\
BAL & $39 / 52(75.0)$ & $13 / 16(81.3)$ & $10 / 19(52.6)$ & $14 / 28(50.0)$ & $76 / 115(66.1)$ \\
CSF & $51 / 55(92.7)$ & $18 / 19(94.7)$ & $13 / 19(68.4)$ & $20 / 28(71.4)$ & $102 / 121(84.3)$ \\
Lymph node & $36 / 53(67.9)$ & $10 / 16(62.5)$ & $12 / 19(63.2)$ & $12 / 28(42.9)$ & $70 / 116(60.3)$ \\
Biopsy material & $38 / 53(71.7)$ & $12 / 17(70.6)$ & $2 / 19(10.5)$ & $12 / 28(42.9)$ & $64 / 117(54.7)$ \\
Bone marrow & $37 / 53(69.8)$ & $12 / 16(75.0)$ & $13 / 19(68.4)$ & $15 / 28(53.6)$ & $77 / 116(66.4)$ \\
Rifampicin drug resistance & $33 / 47(70.2)$ & $11 / 15(73.3)$ & $8 / 19(42.1)$ & $9 / 28(32.1)$ & $61 / 109(56.0)$ \\
Isoniazid drug resistance & $31 / 46(67.4)$ & $9 / 15(60.0)$ & $8 / 19(42.1)$ & $10 / 28(35.7)$ & $58 / 108(53.7)$ \\
\hline
\end{tabular}

BAL, bronchoalveolar lavage; CSF, cerebrospinal fluid; (+), positive; (-), negative.

estimated to be less than $2 \%$ overall by $52.0 \%$ of laboratories responding (79/152).

RAPID MOLECULAR DIAGNOSIS

Laboratories were asked whether they were currently using "molecular tests" for tuberculosis, whether they performed these tests in-house, or whether they sent samples away for analysis. In the United Kingdom, $22.5 \%$ of laboratories (37/165) regularly used molecular tests, but only $2.1 \%$ (3/142) used their own in-house amplification tests.

In practice more than half the laboratories $(51.1 \%, 46 / 90)$ did not send any samples for analysis, $45.6 \%$ (41/90) sent between one and four samples a month, 3.3\% (3/90) sent between five and 14 samples, and no laboratory sent more than 14 samples a month.

Laboratories in England and Wales were asked detailed questions about their perceived clinical need for rapid molecular tests for different primary samples in immunocompetent and immunocompromised patients and to comment on their use of these tests as a function of costs. Tables 2 and 3 illustrate the regional differences in test uptake for immunocompetent and immunocompromised patients.

PERFORMANCE OF PHLS MRU, REGIONAL

REFERENCE CENTRES, AND SMRL

Participating laboratories were asked to grade the performance of the MRU/SMRL/RCM in general, as well as for their provision of technical, scientific, and medical advice, and their specific performance with regard to mycobacterial identification and drug susceptibility testing. Laboratories were asked to grade performance as "very good," "good," "average," "poor," or "bad" in response to the questions posed. Results for the United Kingdom (or for England and Wales alone in some cases) are given in table 4 .
Discussion

The audit was performed to assess the effects of a period of change in the organisation of reference services within the PHLS in England and Wales - that is, the move to a geographical organisation of services and the transfer of the MRU to London in November 1995. It facilitated the formulation of new national reference services and gave client laboratories the opportunity to make their views known at an early stage in planning. This national audit built on earlier audits conducted by the SMRL and by Birmingham.

Overall, $67.2 \%$ of all United Kingdom laboratories submitting mycobacteria responded. Nearly all these laboratories were fully or conditionally CPA accredited (although this question was not asked in the north of England) and take part in the NEQAS external proficiency scheme, but few United Kingdom laboratories $(36.5 \%)$ have specific internal quality control systems.

Laboratories varied in size and on average only $3.3 \%$ of primary samples submitted for mycobacterial diagnosis in 1995 produced a mycobacterial culture from approximately half as many patients - that is, a mean of 1488 specimens producing 49 isolates from 23 patients. Clearly for some laboratories so few samples are being processed for mycobacteria that it would be difficult to maintain expertise and proficiency and it would be logical for these laboratories to send samples to larger centres. As there were 257 laboratories known to be processing samples, it is possible that over 380000 specimens are processed for mycobacterial investigation in the United Kingdom each year.

The majority of laboratories decontaminate sputa and comparable specimens using 4\% $\mathrm{NaOH}$ with or without $\mathrm{N}$-acetyl cysteine (NALC), although in practice several different

Table 4 Mean United Kingdom performance of Public Health Laboratory Service Mycobacterium Reference Unit, regional centres for mycobacteriology, and the Scottish Mycobacteria Reference Laboratory, as assessed by all UK laboratories responding to the questionnaire

\begin{tabular}{llllll}
\hline \multirow{2}{*}{ Question } & \multicolumn{2}{l}{ Response (n (\%)) } & & \\
\cline { 2 - 6 } & Very good & Good & Average & Poor & \multirow{2}{*}{ Bad } \\
\hline Quality of service overall & $57 / 181(31.5)$ & $98 / 181(54.1)$ & $26 / 181(14.4)$ & $0 / 181(0)$ & $0 / 181(0)$ \\
Accuracy of identification & $42 / 116(36.2)^{\star}$ & $62 / 116(53.4)^{\star}$ & $12 / 116(10.3)^{\star}$ & $0 / 116(0)^{\star}$ & $0 / 116(0)$ \\
Speed of identification & $32 / 175(18.3)$ & $79 / 175(45.1)$ & $58 / 175(33.1)$ & $6 / 175(3.4)$ & $0 / 175(0)$ \\
Accuracy/helpfulness of susceptibility tests & $60 / 164(36.6)$ & $84 / 164(51.2)$ & $17 / 164(10.4)$ & $3 / 164(1.8)$ & $0 / 164(0)$ \\
Speed for drug susceptibility testing & $32 / 172(18.6)$ & $84 / 172(48.8)$ & $52 / 172(30.2)$ & $3 / 172(1.7)$ & $1 / 142(0.7)$ \\
Overall advice & $40 / 131(30.5)^{\star}$ & $74 / 131(56.5)^{\star}$ & $16 / 131(12.2)^{\star}$ & $1 / 131(0.8)^{\star}$ & $0 / 131(0)$ \\
Scientific/technical advice & $64 / 161(39.8)$ & $83 / 161(51.6)$ & $14 / 161(8.7)$ & $0 / 161(0)$ & $0 / 161(0)$ \\
Medical/clinical advice & $52 / 140(37.1)$ & $73 / 140(52.1)$ & $13 / 140(9.3)$ & $1 / 140(0.7)$ & $1 / 140(0.7)$ \\
\hline
\end{tabular}

*Question not asked in Scotland; mean for England and Wales alone. 
permutations on the basic protocols have been developed. Solid media, mostly of commercial origin, are used by nearly all laboratories and almost two thirds use a liquid media system. The accelerating use of liquid culture systems, while improving sensitivity and reducing detection times, also poses greater safety hazards and requires modern high quality category 3 containment facilities. ${ }^{9}$ Most laboratories incubated cultures for at least eight weeks and almost one third for up to 12 weeks, which is essential if some slow growing mycobacteria are to be detected. The development of standardised protocols for microscopy, decontamination, and culture is essential for consistent quality diagnosis, particularly if there is sufficient flexibility to accommodate new developments such as automated, continuously monitored culture systems.

Only a minority of laboratories use molecular diagnostic tests and most send samples away for analysis. In practice, over half of the laboratories responding (51.1\%) in England and Wales sent no samples for molecular analysis for tuberculosis and nearly all the remainder $(45.6 \%)$ sent four samples or less each month. In general, laboratories were more likely to use molecular methods for immunocompromised patients than immunocompetent ones, although cerebrospinal fluid would be sent in either case, reflecting the difficulty and uncertainty of diagnosing tuberculous meningitis and its clinical importance in all cases. For nearly all samples, particularly if they came from immunocompromised patients, more laboratories referring to London needed rapid identification of tuberculosis than in other regions, reflecting the high incidence of tuberculosis and immunocompromised patients in London, particularly those who are HIV positive. The decision on whether to send a specimen would be strongly influenced by financial considerations, with a clear reduction in the percentage of laboratories sending specimens as the price increased even when the patient was immunocompromised.

Within England and Wales, 43.9\% (47/107) and $56 \%(61 / 109)$ of laboratories wanted and would use a rapid service for the diagnosis of rifampicin resistance in immunocompetent and immunocompromised patients, respectively, but again the decision would be strongly influenced by cost (data not shown). Samples from immunocompromised patients would have priority. There was a strong regional bias, with significantly more laboratories referring specimens to London and Birmingham.

In response to these findings, additional national molecular amplification diagnostic services were established, including rapid rifampicin drug resistance detection which is offered nationally by the PHLS MRU. In appropriate cases rifampicin resistant tuberculosis can be diagnosed from smear positive sputum in two to three days. Similarly a national service to diagnose tuberculous meningitis has been instituted at the MRU. Logically, the cost of creating the appropriate infrastructure for molecular amplification analysis together with the necessary training and relatively high unit cost for these tests means that these services should be created at a limited number of sites.

Laboratories were keen to receive training and written protocols on microscopy, decontamination, and culture methods. Standardised protocols are being developed within the PHLS, for example, as part of this process.

An important purpose of the audit was to facilitate comment on the mycobacterial services offered and encouragingly, the overall service was rated as "very good" or "good" by $85.6 \%$ of respondents nationally. The quality of the advice given in general, the technical/ scientific advice and the clinical/medical advice was rated as "very good" or "good" by $87 \%$, $91.4 \%$, and $89.2 \%$ of respondents, respectively. Speed of speciation and drug susceptibility testing was perceived as a problem by some laboratories.

It is essential, therefore, for national reference centres to establish their proficiency in international schemes. The PHLS MRU is a World Health Organisation supranational reference laboratory and European coordinating centre within the Global Programme on Drug Resistance. This programme is committed to monitoring and maintaining proficiency internationally. As part of this process, the SMRL and the regional centres for mycobacteriology are now linked to the PHLS MRU as part of a joint commitment to maintain and improve tuberculosis drug susceptibility testing nationally.

In conclusion, the development and implementation of both rapid culture techniques and other rapid diagnostic methods for the diagnosis of tuberculosis in primary specimens is clearly a priority, along with standardised protocols for mycobacteriology.

We would like to thank all the consultant microbiologists and senior technical staff at microbiology laboratories who completed what was a substantial questionnaire. We hope that you have felt the exercise was useful as it has been used to modify practice and plan future developments. Special thanks go to Miss Michaela Emery and Mr Dean Wilkinson for their help in Miss Michaela Emery and Mr Dean Wilkinson for their help in
the distribution of the audit and their assistance in data entry. the distribution of the audit and their assistance in data entry. The audit was funded internally using PHLS resources and in
Scotland by CRAG.

1 Drobniewski F, Pablos-Mendez A, Raviglione MC. Epidemiology of tuberculosis in the world. Semin Resp Crit Care Med 1997;18:419-29.

2 Raviglione MR, Snider DE, Kochi A. Global epidemiology of tuberculosis-morbidity and mortality of a world-wide epidemic. $\mathcal{F A M A}$ 1995;273:220-6.

3 Murray CJL, Styblo K, Rouillon A. Tuberculosis in developing countries: burden, intervention and cost. Bull Int Union Tuber Lung Dis 1990;65:6-24.

4 Sudre P, ten Dam G, Kochi A. Tuberculosis: a global overview of the situation today. Bull WHO 1992;70:149-59.

5 Dolin PJ, Raviglione MC, Kochi A. Global tuberculosis incidence and mortality during 1990-2000. Bull WHO 1994;72:213-20.

6 Raviglione MC, Sudre P, Rieder HL, et al. Secular trends of uberculosis in Western Europe. Bull WHO 1993;71:297306.

7 Kumar D, Watson JM, Charlett A, et al. Tuberculosis in England and Wales in 1993: results of a national survey. Thorax 1997;52:1060-7.

8 Ormerod LP, Charlett A, Gilham C, et al. Geographical distribution of tuberculosis notifications in national surveys of England and Wales in 1988 and 1993: report of the Public Health Laboratory Service/British Thoracic Society/ Department of Health Collaborative group. Thorax 1998; 53:176-81.

9 Heifets LB, Good RC. Current laboratory methods for the diagnosis of tuberculosis. In: Bloom BR, ed. Tuberculosis: pathogenesis, protection and control. Washington DC: American Society for Microbiology, 1994:85-110. 